\title{
Erratum: Estimates for disk and ejecta masses produced in compact binary mergers [Phys. Rev. D 101, 103002 (2020)]
}

Christian J. Krüger@ and Francois Foucart๑

(2) (Received 14 September 2020; published 6 October 2020)

DOI: 10.1103/PhysRevD.102.089901

In Fig. 5, we have to report two corrections for the labels:

(i) The labels at the color bars should be " $M_{\mathrm{dyn}} / M_{b}$ " (rather than " $M_{\mathrm{dyn}}$ ").

(ii) The labels on the y-axes should be " $M_{\mathrm{BH}}\left[M_{\odot}\right]$ " (rather than " $Q$ "). We are grateful to Claudio Barbieri for pointing out this mistake to us.

The plotted data in the graphs themselves are correct and the graph with corrected labels is shown below.
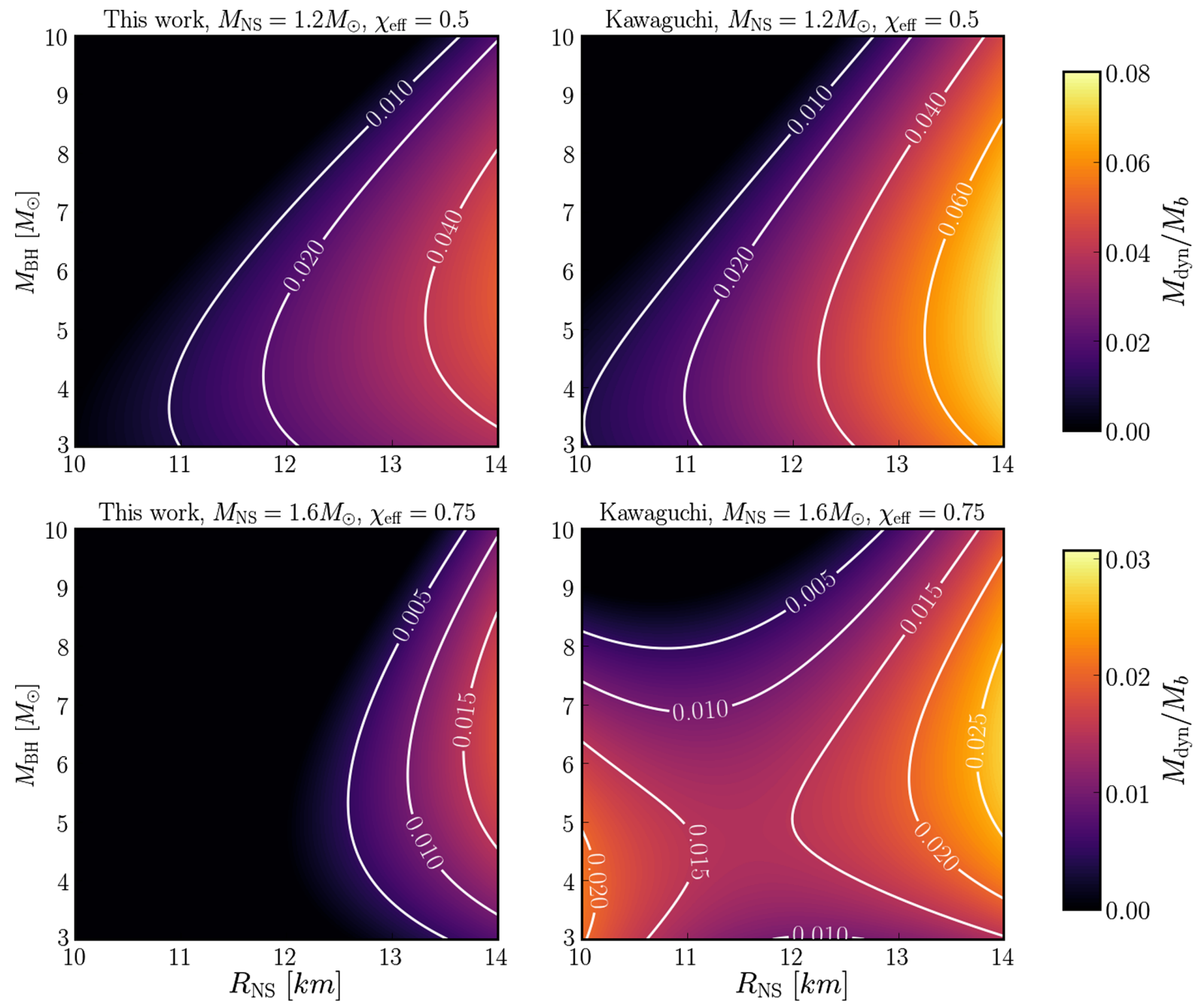

FIG. 5 . 